\title{
Seminomas in the Elderly: A Rare Situation but a Challenging Problem
}

\author{
Daniel Landau" ${ }^{*}$, Michael Landis² and Becca Trieu² \\ ${ }^{1}$ Orlando Health UF Health Cancer Center, USA \\ ${ }^{2}$ University of Central Florida Medical College, USA
}

*Corresponding author: Daniel Landau, University of Florida Cancer Center at Orlando Health, 1400 S Orange Ave MP 760, Orlando, FL 32806, USA, Tel: +1-321-841-7219; Fax: +1-321-841-1296; E-mail: daniel.landau@ orlandohealth.com

\begin{abstract}
While typically a disease of younger men, seminomas do occasionally occur beyond the age of 70. There is less clarity on the treatment for men in this age range. Herein we describe a case of a man in his 80s with aggressive disease, review available literature, and highlight the challenges of treatment men in this age range.
\end{abstract}

Keywords: Seminomas; Germ cell tumor; Triggers; Cisplatin; Chemotherapy

Received Date: January 15, 2019; Accepted Date: January 29, 2019; Published Date: February 05, 2019

\section{Introduction}

Seminoma, a germ cell tumor, is the most common malignancy of the testicle, with a median age of diagnosis at 35 years old. Since 1999-2014, there has been an increased median age for patients diagnosed with testicular germ cell tumors [1]. A typical presentation includes a painless mass with possible swelling and minor pain. Standard therapy includes radical inguinal orchiectomy with chemotherapy for more advanced stages. Radiation may play a role as well. Typical chemotherapeutic regimens for the more advanced disease include the use of cisplatin in a combination with other drugs. Cisplatin, though very effective, produces well documented side effects consisting of nephrotoxicity, neuropathy, and ototoxicity amongst others [2,3].

In rare cases, a seminoma may be diagnosed in elderly patients. Presentation in this patient population can make treatment decisions difficult due to comorbidities of the patients, risk of neutropenia, and the need for close monitoring. In our case we will be discussing an 83 year old male diagnosed with a stage three seminoma. We treated him with a non cisplatin containing regimen that was both efficacious and less toxic than what we would expect for a cisplatin based regimen.

\section{$\mathbf{H} \& \mathbf{P}$}

An 83 year old gentleman presented to my office for evaluation of extensive retroperitoneal adenopathy from his right pelvis to the retrocrural distribution. At that time, he also had hypercalcemia with a total serum calcium of 12.2. Other blood work was unrevealing. Approximately one year prior, the patient was referred to a urologist for a right sided testicular mass. He had an inguinal orchiectomy performed with the pathology showing a classical seminoma with no high-risk features. Further pathologic information could not be obtained as this was done outside of our hospital system and the urologist no longer had access to the slides or report. An abdominal CT scan at that time was reportedly normal. Upon meeting the patient, he had Citation: Daniel Landau, Seminomas in the Elderly; A Rare Situation but a Challenging Problem. J Clin Cases Rep 2(2): 4347. DOI: https://doi.org/10.46619/joccr.2019.2-1037 (C) 2019 Tridha Scholars. 
slight confusion but otherwise was well. Physical exam was unrevealing aside from the slight confusion and there were no palpable lymph nodes. It wasn't immediate clear to us if the confusion was new, but per family members, it was worse than his baseline. He was hard of hearing on exam. Tumor markers including alpha fetoprotein, lactate dehydrogenase, and beta HCG were normal. Aside from the hypercalcemia mentioned above and a baseline creatinine of 2 , other blood work was unrevealing. A biopsy was obtained of a retroperitoneal lymph node. The biopsy was CD117 positive and interpreted as seminoma. The patient required therapy as he had extensive disease with resultant hypercalcemia. The hypercalcemia was attributed directly to his advanced disease. We had extensive conversations with the patient about which chemotherapy to initiate. We ultimately opted to use gemcitabine and carboplatin on a day one and eight out of every 21 day cycle, as we deemed him cisplatin ineligible based on his creatinine level and baseline difficulty hearing. Carboplatin AUC was 5 and Gemcitabine dose 1000/m². This was mirrored from lung cancer regimens. The National Comprehensive Cancer Network guidelines to however list both Gemcitabine and Carboplatin in various combinations as options for treatment of germ cell tumors. After his first cycle, his calcium immediately corrected to normal and his confusion cleared up. He had neutropenia but otherwise minimal complications after the first cycle. Cycle two carried a $25 \%$ dose reduction and was uncomplicated. The remaining four cycles had no complicates and resulted in a rapid and robust response. CT scans including baseline (Figure 1), mid-treatment (Figure 2), and end of treatment (Figure 3) are provided showing the resolution of his adenopathy. The patient at this time is doing well.

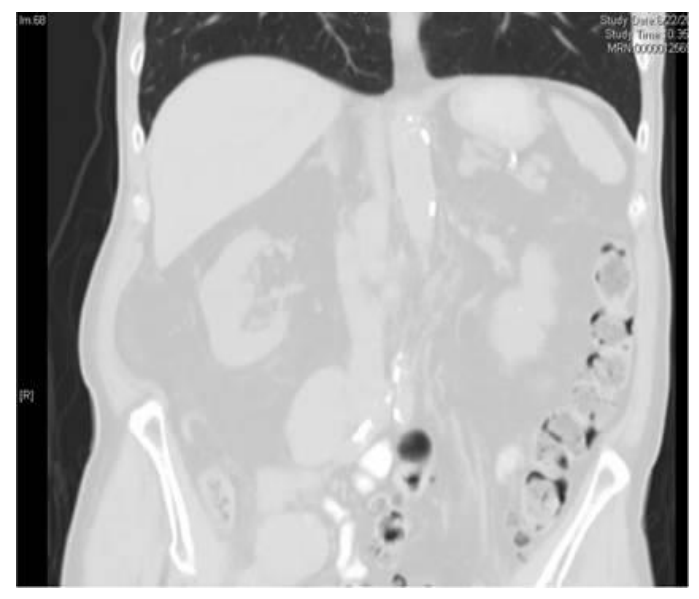

Figure 1: Baseline scan showing extensive adenopathy along the aorta.

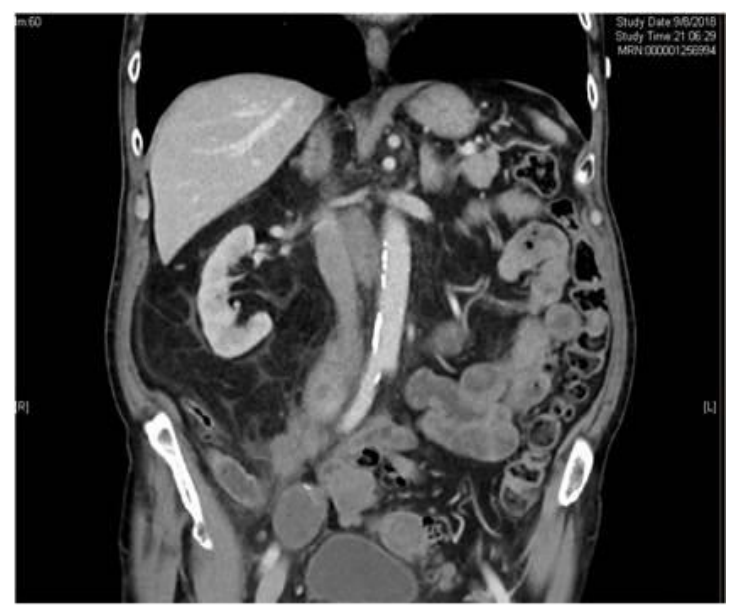

Figure 2: Mid-treatment scan showing decreasing adenopathy compared to figure 1. 


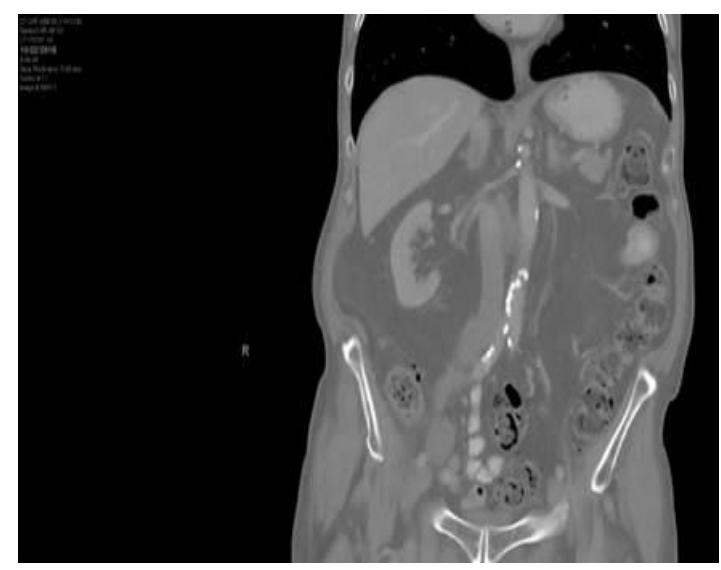

Figure 3: Scan showing resolution of the previously noted adenopathy.

\section{Discussion}

Seminomas have a great prognosis regardless of stage, with a five year survival rate of $66.7 \%$ in patients with stage three diseases [4]. However, this statistic does not directly relate to people far outside the median age of diagnosis where first-line options may not always be available due to comorbidities. Survival rate is highly dependent on both stage and the therapies that are available [4]. For elderly patients with metastatic disease, choosing the proper chemotherapeutic regimen requires carefully weighing the benefits of a first line regimen versus the potentially harmful side effects that a patient of a younger age may be able to tolerate. Given the age and comorbidities, of our patient, he was deemed cisplatin ineligible. Cisplatin has been shown to cause acute kidney injury more often in elderly patients than in younger patients with some studies showing over a $40 \%$ increase in risk [5-7]. Having underlying renal impairment prior to cisplatin use has an additional increased risk for Cisplatin induced nephrotoxicity [8]. In elderly patients with already diminished hearing and potentially decreased sensations, adding a drug such as cisplatin with well-established side effects of ototoxicity and neuropathy could be debilitating to quality of life [3].

Carboplatin and gemcitabine was the chemotherapeutic regimen chosen to treat our patient's seminoma. While carboplatin belongs in the same class as cisplatin, a DNA crosslinking agent, carboplatin is associated with lower risk of AKI [7,9-12]. It has been shown that carboplatin is less neurotoxic and ototoxic than Cisplatin, and our patient didn't experience either of these side effects [13]. On the other hand, an important serious side effect with carboplatin is hematotoxicity especially thrombocytopenia $[9,10,14,15]$. With debatable efficacy between cisplatin and carboplatin, the decision of choosing one over the other is based on their toxicity profiles $[12,15]$. Gemcitabine is a nucleoside analog that halts DNA synthesis. It is commonly used for ovarian cancer, non-small cell lung cancer, pancreatic adenocarcinoma, and cholangiocarcinoma. Literature has shown its efficacy in salvage therapy for germ cell tumors [16-18]. This suggests possible potency against seminoma as first line. A common serious side effect of gemcitabine is hematotoxicity [19-21]. But overall, gemcitabine has been shown to have a favorable side-effect profile especially in the elderly population [19, 22-25]. Efficacy, favorable side effect profile, and higher tolerability in the elderly population are the reasons why carboplatin and gemcitabine were chosen to treat this patient's seminoma.

The successful combination therapy of carboplatin and gemcitabine in our case is of notable importance for many reasons. Seminoma is uncommon in the elderly. Metastatic seminoma requiring aggressive treatment in a patient with renal disease is another distinctive concern. Furthermore, studies using a regimen consisting of carboplatin and gemcitabine for seminomas are 
http://www.tridhascholars.org | April-2019

limited. Although the use for carboplatin in stage one seminoma is well documented in literature, for higher stages of seminoma, there are only a few articles reporting carboplatin being used successfully as monotherapy and combination therapy in elderly patients [26,27]. A new qualitative analysis has shown successful treatment of stage II/III seminoma using carboplatin with higher post-treatment quality of life compared to cisplatin [28]. As for gemcitabine, there is still very limited evidence for its use as first line treatment for seminoma. Our patient responded extremely well to this regimen, and this can serve as a benchmark for future patients with a similar presentation.

\section{Conclusion}

In conclusion, the combination therapy of carboplatin and gemcitabine is shown to successfully treat metastatic seminoma in an elderly patient with multiple comorbidities. Treatment was well tolerated due to its suitable side effect profile for this patient. Due to limited established use of gemcitabine on seminoma, future studies should be done to further investigate similar use.

\section{References}

1. Ghazarian AA, Rusner C, Trabert B, et al. (2018) Testicular cancer among US men aged 50 years and older. Cancer Epidemiology 55: 68-72.

2. Sastry J, Kellie SJ (2005) Severe neurotoxicity, ototoxicity and nephrotoxicity following high-dose cisplatin and amifostine. Pediatric Hematology and Oncology 22(5): 441-445.

3. Arany I, Safirstein RL (2003) Cisplatin nephrotoxicity. In Seminars in Nephrology 23(5): 460-464.

4. Dong W, Gang W, Liu M, et al. (2016) Analysis of the prognosis of patients with testicular seminoma. Oncology Letters 11(2): 1361-1366.

5. Liu JQ, Cai GY, Wang SY, et al. (2018) The characteristics and risk factors for cisplatin-induced acute kidney injury in the elderly. Therapeutics and Clinical Risk Management 14: 1279-1285.

6. Latcha S, Jaimes EA, Patil S, et al. (2016) Long-term renal outcomes after cisplatin treatment. Clinical Journal of the American Society of Nephrology 11 (7): 1173-1179.

7. Duan ZY, Liu JQ, Yin P, et al. (2018) Impact of aging on the risk of platinum-related renal toxicity: A systematic review and meta-analysis. Cancer Treatment Reviews 69: 243-253.

8. Hayati F, Hossainzadeh M, Shayanpour S, et al. (2016) Prevention of cisplatin nephrotoxicity. Journal of Nephropharmacology 5(1): 57-60.

9. Ardizzoni A, Boni L, Tiseo M, (2007) Cisplatin-versus carboplatin-based chemotherapy in first-line treatment of advanced non-small-cell lung cancer: an individual patient data meta-analysis. Journal of the National Cancer Institute 99(11): 847857.

10. Couillard-Montminy V, Gagnon PY, Fortin S, et al. (2017) Effectiveness of adjuvant carboplatin-based chemotherapy compared to cisplatin in non-small cell lung cancer. Journal of Oncology Pharmacy Practice 25(1): 44-51.

11. Klastersky J, Sculier JP, Lacroix H, et al. (1990) A randomized study comparing cisplatin or carboplatin with etoposide in patients with advanced non-small-cell lung cancer: European Organization for Research and Treatment of Cancer Protocol 07861. Journal of Clinical Oncology 8(9): 1556-1562.

12. Santana-Davila R, Szabo A, Arce-Lara C, et al. (2014) Cisplatin versus carboplatin-based regimens for the treatment of patients with metastatic lung cancer. An analysis of veterans health administration data. Journal of Thoracic Oncology 9(5): 702-709. 
http://www.tridhascholars.org | April-2019

13. Amptoulach S, Tsavaris N (2011) Neurotoxicity caused by the treatment with platinum analogues. Chemotherapy Research and Practice 2011: 5.

14. Cheng YJ, Wu R, Cheng ML, et al. (2017) Carboplatin-induced hematotoxicity among patients with non-small cell lung cancer: Analysis on clinical adverse events and drug-gene interactions. Oncotarget 8(19): 32228-32236.

15. de Castria TB, da Silva EM, Gois AF, et al. (2013) Cisplatin versus carboplatin in combination with third-generation drugs for advanced non-small cell lung cancer. Cochrane Database of Systematic Reviews (8): Cd009256.

16. Rashdan S, Einhorn LH (2016) Salvage therapy for patients with germ cell tumor. Journal of Oncology Practice 12(5): 437 443.

17. Mulherin BP, Brames MJ, Einhorn LH (2015) Long-term survival with paclitaxel and gemcitabine for germ cell tumors after progression following high-dose chemotherapy with tandem transplant. American Journal of Clinical Oncology 38(4): 373-376.

18. Oing C, Alsdorf WH, von Amsberg G, et al. (2017) Platinum-refractory germ cell tumors: an update on current treatment options and developments. World Journal of Urology 35(8): 1167-1175.

19. Hiret S, Isambert N, Gomez-Roca C, et al. (2018) Phase I dose-escalation trial of afatinib, an irreversible ErbB family blocker, in combination with gemcitabine or docetaxel in patients with relapsed or refractory solid tumors. Investigational New Drugs 36(6): 1044-1059.

20. Chen H, He R, Shi X, et al. (2018) Meta-analysis on resected pancreatic cancer: a comparison between adjuvant treatments and gemcitabine alone. BMC Cancer 18(1): 1034.

21. Hronek JW, Reed ML (2015) Nursing implications of chemotherapy agents and their associated side effects in patients with pancreatic cancer. Clinical Journal of Oncology Nursing 19(6): 751-757.

22. Tonato M, Mosconi AM, Martin C (1995) Safety profile of gemcitabine. Anti-Cancer Drugs 6: 27-32.

23. Green MR (1996) Gemcitabine safety overview. In Seminars in Oncology 23(5): 32-35.

24. Aapro MS, Martin C, Hatty S (1998) Gemcitabine-a safety review. Anti-Cancer Drugs 9(3): 191-201.

25. Macchini M, Chiaravalli M, Zanon S, et al. (2018) Chemotherapy in elderly patients with pancreatic cancer: efficacy, feasibility and future perspectives. Cancer Treatment Reviews 72: 1-6.

26. Ismaili N, Naciri S, Afqir S, et al. (2008) A rare case of advanced testicular seminoma in a 78-year-old man managed successfully with carboplatin based chemotherapy: a case report. Cases Journal 1(1): 357.

27. Schmoll HJ, Harstrick A, Bokemeyer C, et al. (1993) Single-agent carboplatinum for advanced seminoma a phase II study. Cancer 72(1): 237-243.

28. Milic M, Hall M, Hawkins A, et al. (2019) A qualitative analysis of the impact of carboplatin AUC 10 on physical, work functioning and bone marrow toxicity among seminoma patients-a single-centre experience. In Vivo 33(1): 233-237. 